\title{
On Distributed Reduced-Order Observer-Based Protocol for Linear Multiagent Consensus under Switching Topology
}

\author{
Yan Zhang, Lixin Gao, and Changfei Tong \\ Institute of Intelligent Systems and Decision, Wenzhou University, Zhejiang 325027, China \\ Correspondence should be addressed to Lixin Gao; gao-lixin@163.com
}

Received 21 January 2013; Accepted 18 March 2013

Academic Editor: H. G. Enjieu Kadji

Copyright ( 2013 Yan Zhang et al. This is an open access article distributed under the Creative Commons Attribution License, which permits unrestricted use, distribution, and reproduction in any medium, provided the original work is properly cited.

\begin{abstract}
We discuss linear multiagent systems consensus problem with distributed reduced-order observer-based protocol under switching topology. We use Jordan decomposition method to prove that the proposed protocols can solve consensus problem under directed fixed topology. By constructing a parameter-dependent common Lyapunov function, we prove that the distributed reducedorder observer-based protocol can also solve the continuous-time multi-agent consensus problem under the undirected switching interconnection topology. Then, we investigate the leader-following consensus problem and propose a reduced-order observerbased protocol for each following agent. By using similar analysis method, we can prove that all following agents can track the leader under a class of directed interaction topologies. Finally, the given simulation example also shows the effectiveness of our obtained result.
\end{abstract}

\section{Introduction}

Recently, a great number of researchers pay much attention to the coordination control of the multiagent systems, which have various subject background such as biology, physics, mathematics, information science, computer science, and control science in [1-4]. Consensus problem is one of the most basic problems of the coordination control of the multiagent systems, and the main idea is to design the distributed protocols which enable a group of agents to achieve an agreement on certain quantities of interest. The wellknown early work [1] was done in the control systems community, which gave the theoretical explanation of the consensus behavior of the very famous Vicsek model [2]. Till now, many interesting results for solving similar or generalized consensus problems have been obtained.

The interaction topologies among agents include fixed and switching cases. Fixed topology may be easy to be handled by using eigenvalue decomposition method $[5,6]$. Saber and Murray established a general model for consensus problems of the multiagent systems by introducing Lyapunov method to reveal the contract with the connectivity of the graph theory and the stability of the system in [7]. The
Lyapunov-based approach is often chosen to solve highorder consensus problem $[8,9]$. In most existing works, the dynamics of agents is assumed to be first-, second-, and, sometimes, high-order integrators, and the proposed consensus protocols are based on information of relative states among neighboring agents [10-13]. However, the interacting topology between agents may change dynamically due to the changes of environment, the unreliable communication links and time-delay. It is more difficult to deal with switching interaction topology in mathematics than fixed interaction topology. The common Lyapunov method is fit to probe the switching interaction topology [8]. Too many results have been established for multiagent consensus under switching topology [14-18]. Some other relevant research topics have also been addressed, such as oscillator network [19], cluster synchronization $[20,21]$, mean square consensus [22], fractional-order multiagent systems [23], descriptor multiagent system [24], random networks [25], and time-delay [26].

The leader-following configuration is very useful to design the multiagent systems. Leader-following consensus problems with first-order dynamics under jointly connected interacting topology were investigated by [1]. Hong et al. [8] considered a multiagent consensus problem with 
a second-order active leader and variable interconnection topology. A distributed consensus protocol was proposed for first-order agent with distributed estimation of the general active leader's unmeasurable state variables in [15], while [16] extended the results of [15] to the case of communication delays among agents under switching topology. In [17], the authors considered leader-following problem in the multiagent system with general linear dynamics in both fixed topology case and switching topology case, respectively. The cooperative output regulation of linear multiagent systems can be viewed as a generalization of some results of the leaderfollowing consensus problem of multiagent systems [27, 28].

In many practical systems, the state variables cannot be obtained directly. To achieve the state consensus, the agent has to estimate those unmeasurable state variables by output variables. In [8], the authors proposed a distributed observerbased tracking protocols for each first-order following agent. Under the assumption that the active leader's velocity cannot be measured directly, [29] proposed a distributed observerbased tracking protocol for each second-order followingagent. To track the accelerated motion leader, [30] proposed an observer-based tracking protocol for each second-order follower agent to estimate the acceleration of the leader. A robust adaptive observer based on the response system was constructed to practically synchronize a class of uncertain chaotic systems [31]. In [23], the author proposed an observer-type consensus protocol to the consensus problem for a class of fractional-order uncertain multiagent systems with general linear dynamics. For the multiagent system with general linear dynamics, [32] established a unified framework and proposed an observer-type consensus protocol, and [33] proposed a framework including full state feedback control, observer design, and dynamic output feedback control for leader-following consensus problem. The leader-following consensus problem was investigated under a class of directed switching topologies in [34]. In [35], distributed reducedorder observer-based consensus protocols were proposed for both continuous- and discrete-time linear multiagent systems. Other observer-based previous works include [3638].

Motivated by the previous works, especially by [35], we do some further investigations on the reduced-order observerbased consensus protocol problem which had been studied by [35] under directed fixed interconnection topology. We first correct some errors and propose a new proof of the main result established in the aforementioned paper based on the Jordan decomposition method. Moreover, by constructing a parameter-dependent common Lyapunov function, we prove that the proposed protocol can guarantee the multiagent consensus system to achieve consensus under undirected switching topology. Although the Lyapunov function method is conservative and is not easy to be constructed, it is fit to solve the problem under the switching interconnection topology. We propose distributed protocol to solve leaderfollowing consensus with a little simple modification to the reduced-order observer-based consensus protocol. Similarly, we can prove that all following agents can track the leader under a class of directed interaction topologies. As the special cases, the consensus conditions for balanced and undirected interconnection topology cases can be obtained directly. Although the leader-following consensus problem in this paper has been studied in many papers with the aid of internal model principle, we obtain a low-dimensional controller in our model.

The paper is organized as follows. In Section 2, some notations and preliminaries are introduced. Then, in Section 3 and Section 4, the main results on the consensus stability are obtained for both leaderless and leader-following cases, respectively. Following that, Section 5 provides a simulation example to illustrate the established results, and finally, the concluding remarks are given in Section 6.

\section{Preliminaries}

To make this paper more readable, we first introduce some notations and preliminaries, most of which can be found in [35]. Let $R^{m \times n}$ and $C^{m \times n}$ be the set of $m \times n$ real matrices and complex matrices, respectively. $\operatorname{Re}(\xi)$ denotes the real part of $\xi \in C$. $I$ is the identity matrix with compatible dimension. $A^{T}$ and $A^{H}$ represent transpose and conjugate transpose of matrix $A \in C^{m \times n}$, respectively. $\mathbf{1}_{n}=[1, \ldots, 1]^{T} \in R^{n}$. For symmetric matrices $A$ and $B, A>(\geq) B$ means that $A-B$ is positive (semi-) definite. $\otimes$ denotes the Kronecker product, which satisfies $(A \otimes B)(C \otimes D)=(A C) \otimes(B D)$ and $(A \otimes B)^{T}=$ $A^{T} \otimes B^{T}$. A matrix is said to be Hurwitz stable if all of its eigenvalues have negative real parts.

A weighted digraph is denoted by $\mathscr{G}=\{\mathscr{V}, \mathcal{\varepsilon}, A\}$, where $\mathscr{V}=\left\{v_{1}, v_{2}, \ldots, v_{n}\right\}$ is the set of vertices, $\varepsilon \subset \mathscr{V} \times \mathscr{V}$ is the set of edges, and a weighted adjacency matrix $A=\left[a_{i j}\right]$ has nonnegative elements $a_{i j}$. The set of all neighbor nodes of node $v_{i}$ is defined by $\mathcal{N}_{i}=\left\{j \mid\left(v_{i}, v_{j}\right) \in \varepsilon\right\}$. The degree matrix $D=\left\{d_{1}, d_{2}, \ldots, d_{n}\right\} \in \mathscr{R}^{n \times n}$ of digraph $\mathscr{G}$ is a diagonal matrix with diagonal elements $d_{i}=\sum_{j \in \mathscr{N}_{i}} a_{i j}$. Then, the Laplacian matrix of $\mathscr{G}$ is defined as $L=D-A \in R^{n \times n}$, which satisfies $L \mathbf{1}_{n}=0$. The Laplacian matrix has following interesting property.

Lemma 1 (see [14]). The Laplacian matrix L associated with weighted digraph $\mathscr{G}$ has at least one zero eigenvalue and all of the non-zero eigenvalues are located on the open right half plane. Furthermore, $L$ has exactly one zero eigenvalue if and only if the directed graph $\mathscr{G}$ has a directed spanning tree.

A weighted graph is called undirected graph if for all $\left(v_{i}, v_{j}\right) \in \varepsilon$, we have $\left(v_{j}, v_{i}\right) \in \varepsilon$ and $a_{i j}=a_{j i}$. It is well known that Laplacian matrix of weighted undirected graph is symmetric positive semidefinite, which can be derived from Lemma 1 by noticing the fact that all eigenvalues of symmetric matrix are real and nonnegative. Furthermore, Laplacian matrix $L$ has exactly one zero eigenvalue if and only if the undirected graph $\mathscr{G}$ is connected.

To establish our result, the well-known Schur Complement Lemma is introduced.

Lemma 2 (see [39]). Let $S$ be a symmetric matrix of the partitioned form $S=\left[S_{i j}\right]$ with $S_{11} \in R^{r \times r}, S_{12} \in R^{r \times(n-r)}$, and 
$S_{22} \in R^{(n-r) \times(n-r)}$. Then, $S<0$ if and only if

$$
S_{11}<0, \quad S_{22}-S_{21} S_{11}^{-1} S_{12}<0,
$$

or equivalently,

$$
S_{22}<0, \quad S_{11}-S_{12} S_{22}^{-1} S_{21}<0 .
$$

\section{Multiagent Consensus Problem}

Consider a multiagent system consisting of $N$ identical agents, whose dynamics are modeled by

$$
\dot{x}_{i}=A x_{i}+B u_{i}, \quad y_{i}=C x_{i}, \quad i=1, \ldots, N
$$

where $x_{i} \in R^{n}$ is the agent $i$ 's state, $u_{i} \in R^{p}$ agent $i$ 's control input, and $y_{i} \in R^{q}$ the agent $i$ 's measured output. $A, B$, and $C$ are constant matrices with compatible dimensions. It is assumed that $(A, B)$ is stabilizable, $(A, C)$ is observable, and $C$ has full row rank.

To solve consensus problem, a weighted counterpart of the distributed reduced-order observer-based consensus protocol proposed in [35] for agent $i$ is given as follows:

$$
\begin{aligned}
\dot{v}_{i}= & F v_{i}+G y_{i}+\mathrm{TB} u_{i}, \\
u_{i}= & \kappa K Q_{1} \sum_{j \in \mathcal{N}_{i}(t)} a_{i j}(t)\left(y_{i}-y_{j}\right) \\
& +\kappa K Q_{2} \sum_{j \in \mathcal{N}_{i}(t)} a_{i j}(t)\left(v_{i}-v_{j}\right),
\end{aligned}
$$

where $v_{i} \in R^{n-q}$ is the protocol state, the weight $a_{i j}(t)$ is chosen as

$$
a_{i j}(t)= \begin{cases}\alpha_{i j}, & \text { if agent } i \text { is connected to agent } j \\ 0, & \text { otherwise }\end{cases}
$$

$\kappa$ is the coupling strength, and $F \in R^{(n-q) \times(n-q)}, G \in R^{(n-q) \times q}$, $T \in R^{(n-q) \times n}, Q_{1} \in R^{n \times q}$ and, $Q_{2} \in R^{n \times(n-q)}$ are constant matrices, which will be designed later.

For the multiagent system under consideration, the interconnection topology may be dynamically changing, which is assumed that there are only finite possible interconnection topologies to be switched. The set of all possible topology digraphs is denoted as $\mathcal{S}=\left\{\mathscr{G}_{1}, \mathscr{G}_{2}, \ldots, \mathscr{G}_{M}\right\}$ with index set $\mathscr{P}=\{1,2, \ldots, M\}$. The switching signal $\sigma:[0, \infty) \rightarrow \mathscr{P}$ is used to represent the index of topology digraph; that is, at each time $t$, the underlying graph is $\mathscr{G}_{\sigma(t)}$. Let $0=t_{1}, t_{2}, t_{3}, \ldots$ be an infinite time sequence at which the interconnection graph switches. Certainly, it is assumed that chattering does not occur when the switching interconnection topology is considered. The main objective of this section is to design protocol (4), which is used to solve the consensus problem under switching interconnection topology.
Let $x=\left[x_{1}^{T}, x_{2}^{T}, \ldots, x_{N}^{T}\right]^{T}$ and $v=\left[v_{1}^{T}, v_{2}^{T}, \ldots, v_{N}^{T}\right]^{T}$. Then, after manipulation with combining (3) and (4), the closed-loop system can be expressed as

$$
\begin{aligned}
& \frac{d}{d t}\left[\begin{array}{l}
x \\
v
\end{array}\right] \\
& =\left[\begin{array}{cc}
I_{N} \otimes A+L_{\sigma(t)} \otimes\left(\kappa B K Q_{1} C\right) & L_{\sigma(t)} \otimes\left(\kappa B K Q_{2}\right) \\
I_{N} \otimes(G C)+L_{\sigma(t)} \otimes\left(\kappa T B K Q_{1} C\right) & I_{N} \otimes F+L_{\sigma(t)} \otimes\left(\kappa T B K Q_{2}\right)
\end{array}\right] \\
& \quad \times\left[\begin{array}{c}
x \\
v
\end{array}\right] .
\end{aligned}
$$

We first discuss consensus problem under fixed interconnection topology, which has been investigated by [35]. In this case, the subscript $\sigma(t)$ in closed-loop system (6) should be dropped. Algorithm 3.1 in [35] is slightly modified to present as follows, which is used to choose control parameters in protocol (4).

Algorithm 3. Given $(A, B, C)$ with properties that $(A, B)$ is stabilizable, $(A, C)$ is observable, and $C$ has full row rank $q$, the control parameters in the distributed consensus protocol (4) are selected as follows.

(1) Select a Hurwitz matrix $F \in R^{(n-q) \times(n-q)}$ with a set of desired eigenvalues that contains no eigenvalues in common with those of $A$. Select $G \in \mathrm{R}^{(n-q) \times q}$ randomly such that $(F, G)$ is controllable.

(2) Solve Sylvester equation

$$
T A-F T=G C
$$

to get the unique solution $T$, which satisfies that $\left[\begin{array}{l}C \\ T\end{array}\right]$ is nonsingular. If $\left[\begin{array}{l}C \\ T\end{array}\right]$ is singular, go back to Step 2 to select another $G$, until [ [ $\left.{ }_{T}^{C}\right]$ is nonsingular. Compute matrices $Q_{1} \in$ $R^{n \times q}$ and $Q_{2} \in R^{n \times(n-q)}$ by $\left[Q_{1} Q_{2}\right]=\left[\begin{array}{c}C \\ T\end{array}\right]^{-1}$.

(3) For a given positive definite matrix $Q$, solve the following Riccati equation:

$$
A^{T} P+P A-P B B^{T} P+Q=0
$$

to obtain the unique positive definite matrix $P$. Then, the gain matrix $K$ is chosen by $K=-B^{T} P$.

(4) Select the coupling strength $\kappa \geq 1 /\left(2 \min _{\lambda_{i} \neq 0}\left\{\operatorname{Re}\left(\lambda_{i}\right)\right\}\right)$, where $\lambda_{i}$ is the $i$ th eigenvalue of Laplacian matrix $L$.

Remark 4. According to Theorem 8.M6 in [40], if $A$ and F have no common eigenvalues, then the matrix $\left[\begin{array}{l}C \\ T\end{array}\right]$ is nonsingular only if $(A, C)$ is observable and $(F, G)$ is controllable. Thus, the assumptions that $(A, C)$ is detectable and $(F, G)$ is stabilizable in Algorithm 3.1 of [35] may be questionable. Step 3 of Algorithm 3.1 in [35] is an LMI inequality. Here, we propose the Riccati equation to replace the LMI inequality in Step 3 of the algorithm. If we use the LMI design approach proposed by [35], all our following analysis process is also right as long as we do some sight modification. The Riccati equation has been widely studied in the subsequent centuries and has known an impressive range of applications in control 
theory, which will make the condition expressed in Riccati equation easy to be generalized to other cases such as descriptor multiagent system. If $(A, B)$ is stabilizable and $Q$ is a positive definite matrix, the Riccati equation (8) has a unique positive definite matrix $P$, which can be found in many books such as [41]. On the other hand, it is convenient for us to solve Riccati equation by using Matlab toolbox.

The following theorem is a modified vision of Theorem 3.3 in [35], which is the main result of [35]. To prove Theorem 3.3 in [35], the authors mainly use the following assumption. Let $U \in R^{N \times N}$ be such a unitary matrix that $U^{T} L U=\Lambda=\left[\begin{array}{ll}0 & 0 \\ 0 & \Delta\end{array}\right]$, where the diagonal entries of $\Delta$ are the nonzero eigenvalues of $L$. Unfortunately, this assumption is not right.

Since Laplacian matrix $L$ of directed topology graph $\mathscr{G}$ is not symmetric, it can only be assumed that there exists a unitary matrix $U$ satisfying $U^{H} L U=\left[\begin{array}{ll}0 & * \\ 0 & \Delta\end{array}\right]$, where $\Delta$ is an upper triangular matrix and $*$ is a nonzero row vector (see [39]). Thus, I think the proof in [35] is not strict too. On the other hand, the limit function of $v_{i}(t)$ in Theorem 3.3 of [35] is not right, which should be $T \omega(t)$. Now, we propose a strict proof, which is based on Jordan decomposition and may be easier to be understood. Before giving our proof, we present the theorem as follows.

Theorem 5. For the multiagent system (3) whose interconnection topology graph $\mathscr{G}$ contains a directed spanning tree, the dynamic protocol (4) constructed by Algorithm 3 can solve the consensus problem. Moreover,

$$
\begin{aligned}
x_{i}(t) & \longrightarrow \omega(t) \triangleq\left(r^{T} \otimes e^{A t}\right)\left[\begin{array}{c}
x_{1}(0) \\
\vdots \\
x_{N}(0)
\end{array}\right]=e^{A t} \sum_{j=1}^{N} r_{j} x_{j}(0), \\
v_{i} & \longrightarrow T \omega(t), \quad i=1,2, \ldots, N, \text { as } t \longrightarrow \infty,
\end{aligned}
$$

where $r=\left(r_{1}, r_{2}, \ldots, r_{N}\right)^{T} \in R^{N}$ is a nonnegative vector such that $r^{T} L=0$ and $r^{T} \mathbf{1}=1$.

Proof. By Lemma 1, the assumption that $\mathscr{G}$ contains a directed spanning tree means that zero is a simple eigenvalue of $L$ and all other eigenvalues of $L$ have positive real parts. From Jordan decomposition of $L$, let $S$ be nonsingular matrix such $S^{-1} L S=J=\left[\begin{array}{ll}0 & 0 \\ 0 & J_{1}\end{array}\right]$, where the Jordan matrix $J_{1} \in$ $C^{(N-1) \times(N-1)}$ is an upper triangular matrix and the diagonal entries of $J_{1}$ are the nonzero eigenvalues of $L$.

Let $\bar{x}=\left(S^{-1} \otimes I_{n}\right) x$ and $\bar{v}=\left(S^{-1} \otimes I_{n-q}\right) v$. Then, system (6) can be represented in terms of $\bar{x}$ and $\bar{v}$ as follows:

$\frac{d}{d t}\left[\begin{array}{l}\bar{x} \\ \bar{v}\end{array}\right]$

$$
\begin{aligned}
= & {\left[\begin{array}{cc}
I_{N} \otimes A+J \otimes\left(\kappa B K Q_{1} C\right) & J \otimes\left(\kappa B K Q_{2}\right) \\
I_{N} \otimes(G C)+J \otimes\left(\kappa T B K Q_{1} C\right) & I_{N} \otimes F+J \otimes\left(\kappa T B K Q_{2}\right)
\end{array}\right] } \\
& \times\left[\begin{array}{c}
\bar{x} \\
\bar{v}
\end{array}\right] .
\end{aligned}
$$

Certainly, system (10) can be divided into the following two subsystems: one is

$$
\frac{d}{d t}\left[\begin{array}{c}
\bar{x}^{0} \\
\bar{v}^{0}
\end{array}\right]=\left[\begin{array}{cc}
A & 0 \\
G C & F
\end{array}\right]\left[\begin{array}{c}
\bar{x}^{0} \\
\bar{v}^{0}
\end{array}\right]
$$

and the other one is

$$
\begin{aligned}
& \frac{d}{d t}\left[\begin{array}{l}
\bar{x}^{1} \\
\bar{v}^{1}
\end{array}\right] \\
& =\left[\begin{array}{cc}
I_{N-1} \otimes A+J_{1} \otimes\left(\kappa B K Q_{1} C\right) & J_{1} \otimes\left(\kappa B K Q_{2}\right) \\
I_{N-1} \otimes(G C)+J_{1} \otimes\left(\kappa T B K Q_{1} C\right) & I_{N-1} \otimes F+J_{1} \otimes\left(\kappa T B K Q_{2}\right)
\end{array}\right] \\
& \quad \times\left[\begin{array}{c}
\bar{x}^{1} \\
\bar{v}^{1}
\end{array}\right],
\end{aligned}
$$

where $\bar{x}=\left[\bar{x}^{0 T}, \bar{x}^{1 T}\right]^{T}$ and $\bar{v}=\left[\bar{v}^{0 T}, \bar{v}^{1 T}\right]^{T}$ with $\bar{x}^{0}$ and $\bar{v}^{0}$ being their first $n$ and $n-p$ column, respectively.

Denote $z_{i}(k)$ for agent $i$ as

$$
z_{i}=x_{i}-\sum_{j=1}^{N} r_{j} x_{j}, \quad i=1, \ldots, N .
$$

Obviously, for all $i, j=1,2, \ldots, N, z_{i}=0$ if and only if $x_{i}=x_{j}$; that is, the consensus is achieved. Let $z=\left[\mathrm{z}_{1}{ }^{T}\right.$, $\left.z_{2}{ }^{T}, \ldots, z_{N}^{T}\right]^{T}$. Then, we have $z=\left(\left(I_{N}-1 r^{T}\right) \otimes I_{n}\right) x$. Let $\bar{z}=\left(S^{-1} \otimes I_{n}\right) z$. We know that $\bar{z}=0$ if and only if $z=0$. Since $L S=S J$, the first column of $S$ is right zero eigenvector $w_{r}$ of $L$. Similarly, $S^{-1} L=J S^{-1}$ implies that the first row of $S^{-1}$ is is left zero eigenvector $w_{l}^{T}$. Set $S=\left[w_{r}, S_{1}\right]$ and $S^{-1}=\left[\begin{array}{c}w_{l}^{T} \\ Y_{1}\end{array}\right]$. Due to $S^{-1} S=I$, we have $w_{l}^{T} w_{r}=1, w_{l}^{T} S_{1}=0$, and $Y_{1} w_{r}=0$. Since 1 and $r^{T}$ are the right and left zero eigenvectors of $L$, respectively, and zero is simple eigenvalue of $L$, there exists constant $\alpha \neq 0$ such that $w_{r}=\alpha \mathbf{1}$ and $w_{l}=(1 / \alpha) r$. Then, we can verify directly that

$$
\begin{aligned}
S^{-1} & \left(I_{N}-\mathbf{1} r^{T}\right) S=S^{-1} S-S^{-1} \mathbf{1} r^{T} S=S^{-1} S-S^{-1} w_{r} w_{l}^{T} S \\
\quad= & {\left[\begin{array}{cc}
0 & 0 \\
0 & I_{N-1}
\end{array}\right] . }
\end{aligned}
$$

Thus, we have

$$
\begin{aligned}
\bar{z} & =\left(S^{-1} \otimes I_{n}\right) z=\left(S^{-1} \otimes I_{n}\right)\left(\left(I_{N}-\mathbf{1} r^{T}\right) \otimes I_{n}\right)\left(S \otimes I_{n}\right) \bar{x} \\
& =\left(\left[\begin{array}{cc}
0 & 0 \\
0 & I_{N-1}
\end{array}\right] \otimes I_{n}\right) \bar{x}=\left[\begin{array}{c}
0 \\
\bar{x}^{1}
\end{array}\right],
\end{aligned}
$$

From the previous analysis, we know that $\bar{x}_{1}=0 \Leftrightarrow \bar{z}=0 \Leftrightarrow$ $z=0$. Thus, the stability of system (12) implies that multiagent system (6) can achieve consensus. Let $\bar{T}=\left[\begin{array}{cc}I_{N-1} \otimes I_{n} & 0 \\ -I_{N-1} \otimes T & I_{N-1} \otimes I_{n-q}\end{array}\right]$, which is nonsingular and $\bar{T}^{-1}=\left[\begin{array}{cc}I_{N-1} \otimes I_{n} & 0 \\ I_{N-1} \otimes T & I_{N-1} \otimes I_{n-q}\end{array}\right]$. Let $\zeta=$ $\bar{T}\left[\begin{array}{c}\bar{x}^{1} \\ \bar{v}^{1}\end{array}\right]$. By Step (2) of Algorithm 3, system (12) is equivalent to the following system:

$$
\zeta=\left[\begin{array}{cc}
I_{N-1} \otimes A+J_{1} \otimes(\kappa B K) & J_{1} \otimes\left(\kappa B K Q_{2}\right) \\
0 & \mathrm{I}_{N-1} \otimes F
\end{array}\right] \zeta \triangleq \bar{F} \zeta .
$$


The matrix $\bar{F}$ is block upper triangular matrix with diagonal block matrix entries $A+\kappa \lambda_{i} B K(i=2,3, \ldots, N)$ and $F$. By Step (3) and Step (4) of Algorithm 3, the unique positive definite solution $P$ of Riccati equation (8) satisfies

$$
\begin{aligned}
(A & \left.+\lambda_{i} \kappa B K\right)^{H} P+P\left(A+\lambda_{i} \kappa B K\right) \\
& =-Q+P B B^{T} P-2 \operatorname{Re}\left(\lambda_{i}\right) \kappa P B B^{T} P \leq-Q
\end{aligned}
$$

that is, $A+\kappa \lambda_{i} B K(i=2,3, \ldots, N)$ are stable. Thus, $\bar{F}$ is stable. Moreover, system (12) is asymptotically stable; that is, the consensus problem can be solved by protocol (4).

From the first equation of system (11), we have $\bar{x}^{0}(t)=$ $e^{A t} \bar{x}^{0}(0)=e^{A t}\left(w_{l}^{T} \otimes I_{n}\right) \times\left[x_{1}^{T}(0), x_{2}^{T}(0), \ldots, x_{N}^{T}(0)\right]^{T}$. In addition, the solution of system (6) under fixed topology satisfies $x(t)=\left(S \otimes I_{n}\right) \bar{x}(t)=\left[\alpha \mathbf{1} \otimes I_{n}, S_{1} \otimes I_{n}\right]\left[\begin{array}{c}\bar{x}^{0}(t) \\ \bar{x}^{1}(t)\end{array}\right] \rightarrow$ $\left[\alpha \mathbf{1} \otimes I_{n}, S_{1} \otimes I_{n}\right]\left[\begin{array}{c}\bar{x}^{0}(t) \\ 0\end{array}\right]=\left[\begin{array}{c}\alpha \bar{x}^{0}(t) \\ \vdots \\ \alpha \bar{x}^{0}(t)\end{array}\right]$, as $t \rightarrow \infty$. Thus, $x_{i}(t) \rightarrow \alpha e^{A t}\left(w_{l}^{T} \otimes I_{n}\right)\left[x_{1}^{T}(0), x_{2}^{T}(0), \ldots, x_{N}^{T}(0)\right]^{T}=e^{A t}\left(r^{T} \otimes\right.$ $\left.I_{n}\right)\left[x_{1}^{T}(0), x_{2}^{T}(0), \ldots, x_{N}^{T}(0)\right]^{T}=\left(r^{T} \otimes e^{A t}\right)\left[\begin{array}{c}x_{1}(0) \\ \vdots \\ x_{N}(0)\end{array}\right]$, as $t \rightarrow$ $\infty$. From the second equation of system (11), we have

$$
\begin{aligned}
& \frac{d}{d t}\left(\bar{v}^{0}(t)-T \bar{x}^{0}(t)\right) \\
& \quad=F \bar{v}^{0}(t)+G C \bar{x}^{0}(t)-T A \bar{x}^{0}(t)=F\left(\bar{v}^{0}(t)-T \bar{x}^{0}(t)\right) .
\end{aligned}
$$

Since $F$ is Hurwitz stable, we know that $\lim _{t \rightarrow \infty}\left(\bar{v}^{0}(t)-\right.$ $\left.T \bar{x}^{0}(t)\right)=0$. Noticing that $v=\left(S \otimes I_{n-q}\right) \bar{v}$, we can also obtain $v_{i}(t) \rightarrow \alpha \bar{v}^{0}(t)$, as $t \rightarrow \infty$. Thus, we have $v_{i}(t) \rightarrow \alpha T \bar{x}^{0}(t)=$ $T \omega(t)$, as $t \rightarrow \infty$. The proof is now completed.

Next, we probe the consensus problem under switching interconnection topology. For the switching interconnection topology case, we always assume that all interconnection topology graphs $\mathscr{G}_{i}, i \in \mathscr{P}$ are undirected and connected. Choose an orthogonal matrix with form $U=\left[(1 / \sqrt{N}) \mathbf{1}, U_{1}\right]$ with $U_{1} \in R^{N \times(N-1)}$. Noticing that the Laplacian matrix $L_{i}$ of $\mathscr{G}_{i}(i \in \mathscr{P})$ is symmetric and $L_{i} \mathbf{1}=0$, we have

$$
U^{T} L_{i} U=\left(\begin{array}{cc}
0 & 0 \\
0 & \widetilde{L}_{1 i}
\end{array}\right):=\widetilde{L}_{i}
$$

where $\widetilde{L}_{1 i}$ is an $(N-1) \times(N-1)$ symmetric matrix. Since all $\mathscr{G}_{i}$ are undirected and connected, $L_{i}$ is positive semidefinite and $\widetilde{L}_{1 i}$ is positive definite.

Then, we can define

$$
\begin{aligned}
& \bar{\lambda}=\min _{i \in \mathscr{P}}\left\{\lambda_{2}\left(L_{i}\right) \mid \mathscr{G}_{i} \text { is undirected and connected }\right\}>0, \\
& \tilde{\lambda}=\max _{i \in \mathscr{P}}\left\{\lambda_{\max }\left(L_{i}\right) \mid \mathscr{G}_{i} \text { is undirected and connected }\right\}>0,
\end{aligned}
$$

where $\lambda_{2}\left(L_{i}\right)$ is the second small eigenvalue of $L_{i}$. Since $\mathscr{P}$ is finite set, $\bar{\lambda}$ and $\tilde{\lambda}$ are fixed and positive.

To measure the disagreement of $x_{i}(t)$ to the average state of all agents, denote $z_{i}(t)$ for agent $i$ as

$$
z_{i}=x_{i}-\frac{1}{N} \sum_{j=1}^{N} x_{j}
$$

Obviously, $z_{i}=0$ for any $i=1,2, \ldots, N$ if and only if $x_{i}=x_{j}$, for any $i, j=1,2, \ldots, N$; that is, the consensus is achieved. Let $z=\left[z_{1}^{T}, z_{2}^{T}, \ldots, z_{N}^{T}\right]^{T}$. Then, we have

$$
z=\left(L_{o} \otimes I_{n}\right) x
$$

where

$$
L_{o}=I_{N}-\frac{1}{N} \mathbf{1}_{N} \mathbf{1}_{N}^{T}
$$

which satisfies $L_{o} \mathbf{1}_{N}=0$. It can be verified that $U^{T} \mathbf{1}_{N}=$ $[\sqrt{N}, 0, \ldots, 0]^{T}$, from which we have

$$
U^{T} L_{o} U=U^{T} U-\frac{1}{N} U^{T} \mathbf{1 1}^{T} U=\left[\begin{array}{cc}
0 & 0 \\
0 & I_{N-1}
\end{array}\right] \triangleq \widetilde{L}_{o} .
$$

Let $\tilde{x}=\left(U^{T} \otimes I_{n}\right) x, \widetilde{v}=\left(U^{T} \otimes I_{n-q}\right) v$, and $\widetilde{z}=\left(U^{T} \otimes\right.$ $\left.I_{n}\right) z$. Then, system (6) can be expressed in terms of $\tilde{x}$ and $\widetilde{v}$ as follows:

$$
\begin{aligned}
& \frac{d}{d t}\left[\begin{array}{c}
\tilde{x} \\
\tilde{v}
\end{array}\right] \\
& =\left[\begin{array}{cc}
I_{N} \otimes A+\widetilde{L}_{\sigma(t)} \otimes\left(\kappa B K Q_{1} C\right) & \widetilde{L}_{\sigma(t)} \otimes\left(\kappa B K Q_{2}\right) \\
I_{N} \otimes(G C)+\widetilde{L}_{\sigma(t)} \otimes\left(\kappa T B K Q_{1} C\right) & I_{N} \otimes F+\widetilde{L}_{\sigma(t)} \otimes\left(\kappa T B K Q_{2}\right)
\end{array}\right] \\
& \quad \times\left[\begin{array}{c}
\tilde{x} \\
\tilde{v}
\end{array}\right] .
\end{aligned}
$$

Similarly, system (25) can be divided into the following two subsystems:

$$
\frac{d}{d t}\left[\begin{array}{c}
\widetilde{x}^{0} \\
\widetilde{v}^{0}
\end{array}\right]=\left[\begin{array}{cc}
A & 0 \\
G C & F
\end{array}\right]\left[\begin{array}{c}
\tilde{x}^{0} \\
\widetilde{v}^{0}
\end{array}\right]
$$

$$
\begin{aligned}
& \frac{d}{d t}\left[\begin{array}{c}
\tilde{x}^{1} \\
\tilde{v}^{1}
\end{array}\right] \\
& =\left[\begin{array}{cc}
I_{N-1} \otimes A+\widetilde{L}_{1 \sigma(t)} \otimes\left(\kappa B K Q_{1} C\right) & \widetilde{L}_{1 \sigma(t)} \otimes\left(\kappa B K Q_{2}\right) \\
I_{N-1} \otimes(G C)+\widetilde{L}_{1 \sigma(t)} \otimes\left(\kappa T B K Q_{1} C\right) & I_{N-1} \otimes F+\widetilde{L}_{1 \sigma(t)} \otimes\left(\kappa T B K Q_{2}\right)
\end{array}\right] \\
& \quad \times\left[\begin{array}{c}
\tilde{x}^{1} \\
\widetilde{v}^{1}
\end{array}\right],
\end{aligned}
$$

where $\tilde{x}=\left[\widetilde{x}^{0 T}, \tilde{x}^{1 T}\right]^{T}$ and $\widetilde{v}=\left[\widetilde{v}^{0 T}, \widetilde{v}^{1 T}\right]^{T}$ with $\widetilde{x}^{0}$ and $\widetilde{v}^{0}$ being their first $n$ and $n-p$ columns, respectively.

We know that $\widetilde{z}=0$ if and only if $z=0$. On the other hand, we have

$$
\begin{aligned}
\widetilde{z} & =\left(U^{T} \otimes I_{n}\right) z=\left(U^{T} \otimes I_{n}\right)\left(L_{o} \otimes I_{n}\right) x \\
& =\left(\widetilde{L}_{o} \otimes I_{n}\right) \tilde{x}=\left[\begin{array}{c}
0 \\
\tilde{x}^{1}
\end{array}\right],
\end{aligned}
$$

from which we know that $\tilde{x}^{1}=0$ is equivalent to $z=0$. 
Thus, the stability of the switching system (27) implies that multiagent system (6) can achieve consensus.

Denote that $\xi=\left[\begin{array}{cc}I_{N-1} \otimes I_{n} & 0 \\ -I_{N-1} \otimes T & I_{N-1} \otimes I_{n-q}\end{array}\right]\left[\begin{array}{l}\tilde{x}^{1} \\ \widetilde{v}^{1}\end{array}\right]$. By Step (2) of Algorithm 3, system (27) is equivalent to the following switching system:

$$
\dot{\xi}=\widetilde{F}_{\sigma(t)} \xi
$$

where

$$
\widetilde{F}_{\sigma(t)}=\left[\begin{array}{cc}
I_{N-1} \otimes A+\widetilde{L}_{1 \sigma(t)} \otimes(\kappa B K) & \widetilde{L}_{1 \sigma(t)} \otimes\left(\kappa B K Q_{2}\right) \\
0 & I_{N-1} \otimes F
\end{array}\right] .
$$

Next, we investigate consensus problem of multiagent system under switching interconnection topology based on convergence analysis of the switching system (29) and present our main result as follows.

Theorem 6. For the multiagent system (3) whose interconnection topology graph $\mathscr{G}_{\sigma(t)}$ associated with any interval $\left[t_{j}, t_{j+1}\right)$ is assumed to be undirected and connected, suppose that the parameter matrices $F, G, T, K, Q_{1}$, and $Q_{2}$ used in control protocol (4) are constructed by Steps (1)-(3) of Algorithm 3 and the coupling strength $\kappa$ is satisfied as

$$
\kappa \geq \frac{1}{2 \bar{\lambda}}
$$

Then, the distributed control protocol (4) can guarantee that the multiagent system achieves consensus from any initial condition. Moreover,

$$
\begin{gathered}
x_{i}(t) \longrightarrow \omega(t) \triangleq e^{A t}\left[\frac{1}{N} \sum_{j=1}^{N} x_{j}(0)\right], \\
v_{i}(t) \longrightarrow T \omega(t), \quad i=1,2, \ldots, N, \text { as } t \longrightarrow \infty .
\end{gathered}
$$

Proof. Till now, we know that the multiagent system achieves consensus if the state $\xi$ of systems (29) satisfies $\lim _{t \rightarrow \infty} \xi=$ 0 . Although system (29) is switching in $[0, \infty)$, it is timeinvariant in any interval $\left[t_{i}, t_{i+1}\right)$. Assume that $\sigma(t)=p$, which belongs to $\mathscr{P}$. Since $\mathscr{G}_{p}$ is undirected and connected, $\widetilde{L}_{1 p}$ is positive definite. Let $U_{p}$ be an orthogonal matrix such that

$$
U_{p} \widetilde{L}_{1 p} U_{p}^{T}=\Lambda_{p} \triangleq \operatorname{diag}\left\{\lambda_{1 p}, \lambda_{2 p}, \ldots, \lambda_{(N-1)_{p}}\right\},
$$

where $\lambda_{i p}$ is $i$ th eigenvalue of matrix $\widetilde{L}_{1 p}$. The unique positive definite solution $P>0$ of Riccati equation (8) satisfies

$$
\begin{aligned}
(A & \left.+\lambda_{i p} \kappa B K\right)^{T} P+P\left(A+\lambda_{i p} \kappa B K\right) \\
& =-Q+P B B^{T} P-2 \lambda_{i p} \kappa P B B^{T} P \leq-Q,
\end{aligned}
$$

from which we can obtain

$$
\begin{aligned}
& {\left[I \otimes A+\Lambda_{p} \otimes(\kappa B K)\right]^{T}(I \otimes P)+(I \otimes P)} \\
& \quad \times\left[I \otimes A+\Lambda_{p} \otimes(\kappa B K)\right] \leq-I \otimes Q<0 .
\end{aligned}
$$

Pre- and postmultiplying inequality (63) by $U_{p} \otimes I$ and its transpose, respectively, we get

$$
\begin{aligned}
& {\left[I \otimes A+\widetilde{L}_{1 p} \otimes(\kappa B K)\right]^{T}(I \otimes P)+(I \otimes P)} \\
& \quad \times\left[I \otimes A+\widetilde{L}_{1 p} \otimes(\kappa B K)\right] \leq-I \otimes Q<0 .
\end{aligned}
$$

In addition, for stable matrix $F$, there exist positive definite matrices $\bar{Q}$ and $\bar{P}$ satisfying the Lyapunov equation

$$
F^{T} \bar{P}+\bar{P} F=-\bar{Q},
$$

or equivalently,

$$
\begin{aligned}
& \left(I_{N-1} \otimes F\right)^{T}\left(I_{N-1} \otimes \bar{P}\right)+\left(I_{N-1} \otimes \bar{P}\right)\left(I_{N-1} \otimes F\right) \\
& \quad=-\left(I_{N-1} \otimes \bar{Q}\right) .
\end{aligned}
$$

Consider the parameter-dependent Lyapunov function for dynamic system (29)

$$
V(\xi(t))=\xi(t)^{T} \widetilde{P} \xi(t),
$$

where matrix $\widetilde{P}$ has the form

$$
\widetilde{P}=\left(\begin{array}{cc}
\frac{1}{\omega} I \otimes P & 0 \\
0 & I \otimes \bar{P}
\end{array}\right)
$$

with positive parameter $\omega$. In interval $\left[t_{i}, t_{i+1}\right)$, the time derivative of this Lyapunov function along the trajectory of system (29) is

$$
\frac{d}{d t} V(\xi)=\xi^{T}\left(\widetilde{F}_{\sigma}^{T} \widetilde{P}+\widetilde{P} \widetilde{F}_{\sigma}\right) \xi \triangleq \xi^{T} \widetilde{Q}_{\sigma} \xi
$$

where

$$
\begin{gathered}
\widetilde{Q}_{\sigma}=\left(\begin{array}{cc}
\frac{1}{\omega} \widetilde{Q}_{1 \sigma} & \frac{1}{\omega} \widetilde{L}_{1 \sigma(t)} \otimes\left(\kappa P B K Q_{2}\right) \\
\frac{1}{\omega} \widetilde{L}_{1 \sigma(t)}^{T} \otimes\left(\kappa P B K Q_{2}\right)^{T} & I \otimes\left(F^{T} \bar{P}+\bar{P} F^{T}\right)
\end{array}\right), \\
\widetilde{Q}_{1 \sigma}=\frac{1}{\omega}\left[\left(I \otimes A+\widetilde{L}_{1 \sigma(t)} \otimes(\kappa B K)\right)^{T}(I \otimes P)\right. \\
\left.+(I \otimes P)\left(I \otimes A+\widetilde{L}_{1 \sigma(t)} \otimes(\kappa B K)\right)\right] .
\end{gathered}
$$

From (64) and (65), we have

$$
\widetilde{Q}_{\sigma} \leq\left(\begin{array}{cc}
-\frac{1}{\omega} I \otimes Q & \frac{1}{\omega} \widetilde{L}_{1 \sigma(t)} \otimes\left(\kappa P B K Q_{2}\right) \\
\frac{1}{\omega} \widetilde{L}_{1 \sigma(t)}^{T} \otimes\left(\kappa P B K Q_{2}\right)^{T} & -I \otimes \bar{Q}
\end{array}\right) .
$$

Since the constant $\omega$ can be chosen large enough to satisfy

$$
\omega>\tilde{\lambda}^{2} \kappa^{2} \lambda_{\max }\left(Q^{-1}\left(P B K Q_{2}\right)^{T} \bar{Q}^{-1}\left(P B K Q_{2}\right)\right),
$$


this implies that

$$
\begin{aligned}
-\frac{1}{\omega} & (I \otimes Q)+\frac{1}{\omega^{2}}\left[\widetilde{L}_{1 \sigma(t)} \otimes\left(\kappa P B K Q_{2}\right)\right] \\
& \times\left(I \otimes \bar{Q}^{-1}\right)\left[\widetilde{L}_{1 \sigma(t)}^{T} \otimes\left(\kappa P B K Q_{2}\right)^{T}\right]<0 .
\end{aligned}
$$

According to Lemma 2, we know that $\widetilde{Q}_{\sigma}$ is positive definite. Because there are only finite interconnection topology graphs to be switched, we know that system (29) is asymptotically stable; that is, system (6) achieves consensus. Similarly, we can prove that

$$
\begin{gathered}
x_{i}(t) \longrightarrow \omega(t) \triangleq e^{A t}\left[\frac{1}{N} \sum_{j=1}^{N} x_{j}(0)\right], \\
v_{i} \longrightarrow T \omega(t), \quad i=1,2, \ldots, N, \text { as } t \longrightarrow \infty .
\end{gathered}
$$

The proof is now completed.

Remark 7. Here, the topological graph is assumed to be undirected for convenience. If all graphs $\mathscr{G}_{\sigma(t)}$ are directed and balanced with a directed spanning tree, we also have

$$
U^{T} L_{\sigma}(t) U=\left(\begin{array}{cc}
0 & 0 \\
0 & \widetilde{L}_{1 \sigma(t)}
\end{array}\right)
$$

where $\widetilde{L}_{1 \sigma(t)}$ is positive definite (see [18]). Define

$$
\begin{array}{r}
\bar{\lambda}=\min _{l \in \mathscr{P}}\left\{\frac{1}{2} \lambda_{2}\left(L_{l}^{T}+L_{l}\right) \mid \mathscr{G}_{l}\right. \text { is balanced and } \\
\text { has a directed spanning tree }\}>0,
\end{array}
$$

where $\lambda_{2}\left(L_{l}^{T}+L_{l}\right)$ is the second small eigenvalue of $L_{l}^{T}+L_{l}$. Following the similar line to analyze the directed topology as Theorem 11 in the next section, it is not difficult to establish similar condition to guarantee that the multiagent system achieves consensus.

\section{Multiagent Consensus Problem with a Leader}

In this section, we consider the multiagent system consisting of $N$ identical agent and a leader. The dynamics of the following agents are described by system (3), and the dynamics of the leader are given as

$$
\dot{x}_{0}=A x_{0}, \quad y_{0}=C x_{0}, \quad x_{0} \in R^{n}, y_{0} \in R^{q},
$$

where $x_{0}$ is the state of the leader, and $y_{0}$ is the measured output of the leader.

Our aim is to construct the distributed control protocol for each agent to track the leader; that is, $x_{i} \rightarrow x_{0}, t \rightarrow \infty$ for any $i=1,2, \ldots N$. To this end, we propose a reduced-order observer-based consensus protocol for each agent as follows:

$$
\begin{aligned}
\dot{v}_{i}= & F v_{i}+G y_{i}+T B u_{i}, \\
u_{i}= & \kappa K Q_{1}\left[\sum_{j \in \mathcal{N}_{i}(t)} a_{i j}(t)\left(y_{i}-y_{j}\right)+d_{i}(t)\left(y_{i}-y_{0}\right)\right] \\
& +\kappa K Q_{2}\left[\sum_{j \in \mathcal{N}_{i}(t)} a_{i j}(t)\left(v_{i}-v_{j}\right)+d_{i}(t)\left(v_{i}-T x_{0}\right)\right],
\end{aligned}
$$

where $v_{i} \in R^{n-q}$ is the protocol state, $\kappa>0$ is the coupling strength, $a_{i j}(t)$ is chosen by (5), and $d_{i}(t)$ is chosen by

$d_{i}(t)= \begin{cases}\beta_{i}, & \text { if agent } i \text { is connected to the leader at time } t \\ 0, & \text { otherwise }\end{cases}$

where $\beta_{i}$ is positive connected weight of edge $(i, 0)$.

Remark 8. The leader's dynamics is only based on itself, but its system matrices are the same as all following agents. In [17], the authors investigated this leader-following consensus problem by using the distributed state feedback control protocol. In this paper, we will solve the problem via the distributed reduced-order observer-based protocol (50), which needs to be assumed that only the neighbors of leader can obtain the state information of the leader.

In what follows, the digraph $\widehat{\mathscr{G}}$ of order $N+1$ is introduced to model interaction topology of the leader-following multiagent system, whose nodes $v_{i}, i=1,2, \ldots, N$, are used to label $N$ following agents and $v_{0}$ is labeled leader. In fact, $\widehat{\mathscr{G}}$ contains graph $\mathscr{G}$, which models the topology relation of these $N$ followers, and $v_{0}$ with the directed edges from some agents to the leader describes the topology relation among all agents. Node $v_{0}$ is said to be globally reachable, if there is a directed path from every other node to node $v_{0}$ in digraph $\widehat{\mathscr{G}}$.

Let $L_{\sigma(t)}$ be the Laplacian matrix of the interaction graph $\mathscr{G}_{\sigma(t)}$, and let $B_{\sigma(t)}$ be an $N \times N$ diagonal matrix whose $i$ th diagonal element is $d_{i}(t)$ at time $t$. For convenience, denote that $H_{\sigma(t)}=L_{\sigma(t)}+B_{\sigma(t)}$. The matrix $H$ has the following property.

Lemma 9 (see [9]). Matrix $H=L+B$ is positive stable if and only if node 0 is globally reachable in $\widehat{\mathscr{G}}$.

Let $\varepsilon_{i}=x_{i}-x_{0}$, and $\bar{\varepsilon}_{i}=v_{i}-T x_{0}$. Then, the dynamics of $\varepsilon_{i}$ and $\bar{\varepsilon}_{i}$ are described as follows:

$$
\begin{aligned}
\dot{\varepsilon}_{i}= & A \varepsilon_{i}+\kappa B K Q_{1} C \sum_{j \in N_{i}(t)} a_{i j}(t)\left[\left(\varepsilon_{i}-\varepsilon_{j}\right)+d_{i}(t) \varepsilon_{i}\right] \\
& +\kappa K Q_{2}\left[\sum_{j \in N_{i}(t)} a_{i j}(t)\left(\bar{\varepsilon}_{i}-\bar{\varepsilon}_{j}\right)+d_{i}(t)\left(\bar{\varepsilon}_{i}\right)\right],
\end{aligned}
$$




$$
\begin{aligned}
\dot{\bar{\varepsilon}}_{i}= & \dot{v}_{i}-T \dot{x}_{0}=F v_{i}+G C x_{i}+\kappa B K Q_{1} C \\
& \times\left[\sum_{j \in N_{i}(t)} a_{i j}\left(x_{i}-x_{j}\right)+d_{i}(t)\left(x_{i}-x_{0}\right)\right]+\kappa K Q_{2} \\
& \times\left[\sum_{j \in N_{i}(t)} a_{i j}\left(v_{i}-v_{j}\right)+d_{i}(t)\left(v_{i}-T x_{0}\right)\right]-T A x_{0} .
\end{aligned}
$$

According to (7) and (53), we can obtain

$$
\begin{aligned}
\dot{\bar{\varepsilon}}_{i}= & F \bar{\varepsilon}_{i}+G C \varepsilon_{i}+\kappa B K Q_{1} C \\
& \times\left[\sum_{j \in N_{i}(t)} a_{i j}(t)\left(\varepsilon_{i}-\varepsilon_{j}\right)+d_{i}(t) \varepsilon_{i}\right] \\
& +\kappa K Q_{2}\left[\sum_{j \in N_{i}(t)} a_{i j}(t)\left(\bar{\varepsilon}_{i}-\bar{\varepsilon}_{j}\right)+d_{i}(t)\left(\bar{\varepsilon}_{i}\right)\right] .
\end{aligned}
$$

Let $\varepsilon=\left(\varepsilon_{1}^{T}, \varepsilon_{2}^{T}, \ldots, \varepsilon_{N}^{T}\right)^{T}, \bar{\varepsilon}=\left(\bar{\varepsilon}_{1}^{T}, \bar{\varepsilon}_{2}^{T}, \ldots, \bar{\varepsilon}_{N}^{T}\right)^{T}$, and $\xi^{T}=$ $\left(\varepsilon^{T}, \bar{\varepsilon}\right)^{T}$. From (52) and (54), the error dynamics can be represented as

$$
\dot{\xi}=\left[\begin{array}{cc}
I_{N} \otimes A+H_{\sigma} \otimes\left(\kappa B K Q_{1} C\right) & H_{\sigma} \otimes\left(\kappa B K Q_{2}\right) \\
I_{N} \otimes(G C)+H_{\sigma} \otimes\left(\kappa T B K Q_{1} C\right) & I_{N} \otimes F+H_{\sigma} \otimes\left(\kappa T B K Q_{2}\right)
\end{array}\right] \xi .
$$

Let $\eta=\left[\begin{array}{cc}I_{N} \otimes I_{n} & 0 \\ -I_{N} \otimes T & I_{N} \otimes I_{n-q}\end{array}\right] \xi$. Similarly, system (55) is equivalent to the following switching system:

$$
\dot{\eta}=\widehat{F}_{\sigma(t)} \eta
$$

where

$$
\widehat{F}_{\sigma(t)}=\left[\begin{array}{cc}
I_{N} \otimes A+H_{\sigma} \otimes(\kappa B K) & H_{\sigma} \otimes\left(\kappa B K Q_{2}\right) \\
0 & I_{N} \otimes F
\end{array}\right]
$$

From the previous transformation, we know that the stability of error system (29) means that all following agents can track the leader. First, we consider fixed topology case and give the result as follows.

Theorem 10. For the leader-following multiagent systems (3) and (49), whose interconnection topology graph $\widehat{\mathscr{G}}$ is fixed and has a globally reachable node $v_{0}$, suppose that the parameter matrices $F, G, T, K, Q_{1}$, and $Q_{2}$ in the dynamic protocol (50) are constructed by Steps (1)-(3) of Algorithm 3 and the coupling strength $\kappa$ is satisfied as

$$
\kappa \geq \frac{1}{2 \min _{i}\left\{\operatorname{Re}\left(\lambda_{i}(H)\right)\right\}} .
$$

Then, the distributed control protocol (50) guarantees that all following agents can track the leader from any initial condition.

Proof. Of course, $\sigma(t)$ in dynamical equation can also be removed. According to Lemma 9, we know that $H$ is positive stable; that is, all eigenvalues $\lambda_{i}(H)$ of $H$ satisfy $\operatorname{Re}\left(\lambda_{i}(H)\right)>0$.
Similarly, we can do similarity transformation to matrix $\widehat{F}$, which can be similar to block upper triangle matrix with diagonal block matrix entries $A+\kappa \lambda_{i}(H) B K$ and $F$. Based on Step (3) of Algorithm 3 and (58), we know that $A+\kappa \lambda_{i}(H) B K$ is stable. Thus, the error system $\dot{\eta}=\widehat{F} \eta$ is stable.

Next, we probe the leader-following consensus problem under switching interconnection topology. Unlike undirected switching topology assumption in the previous section, we assume that the interconnection topology switches in a class of directed topologies. For convenience, the interconnection topology switches in finite set, which is defined by

$$
\begin{gathered}
\Gamma=\left\{\widehat{\mathscr{G}}_{i} \mid v_{0}\right. \text { is globally reachable node } \\
\text { in graph } \widehat{\mathscr{G}}_{i} \text { and } H^{T}\left(\widehat{\mathscr{G}}_{i}\right)+H\left(\widehat{\mathscr{G}}_{i}\right) \\
\text { is positive definite, } \left.i \in \mathscr{P}_{0}\right\}
\end{gathered}
$$

with index set $\mathscr{P}_{0}=\left\{1,2, \ldots, M_{0}\right\}$.

Therefore, define

$$
\widehat{\lambda}:=\min _{\widehat{\mathscr{G}} \in \Gamma}\left\{\lambda\left(H^{T}(\widehat{\mathscr{G}})+H(\widehat{\mathscr{G}})\right)\right\} .
$$

Noticing that the set $\Gamma$ is finite set and $H^{T}(\widehat{\mathscr{G}})+H(\widehat{\mathscr{G}})$ is positive definite, we know that $\hat{\lambda}$ is well defined, which is positive and depends directly on the constants, all constants $a_{i j}$ and $\beta_{i}(i, j=1,2, \ldots, n)$ given in (5) and (51).

Theorem 11. For the leader-following multiagent system (3) and (49), whose interconnection topology graph $\widehat{\mathscr{G}}_{\sigma(t)}$ associated with any interval $\left[t_{j}, t_{j+1}\right)$ is assumed to have a globally reachable node $v_{0}$, suppose that the parameter matrices $F, G$, $T, K, Q_{1}$, and $Q_{2}$ used in control protocol (50) are constructed by Steps (1)-(3) of Algorithm 3 and the coupling strength $\kappa$ is satisfied as

$$
\kappa \geq \frac{1}{2 \widehat{\lambda}} .
$$

Then, the distributed control protocol (50) guarantees that all following agents can track the leader from any initial condition.

Proof. In time interval $\left[t_{i}, t_{i+1}\right)$, assume that $\sigma(t)=p \epsilon$ $\mathscr{P}_{0}$. There exists an orthogonal transformation $U_{p}$ such that $U_{p}\left(H_{p}^{T}+H_{p}\right) U_{p}^{T}$ is a diagonal matrix $\Lambda_{p}=\operatorname{diag}\left\{\lambda_{1 p}, \lambda_{2 p}, \ldots\right.$, $\left.\lambda_{n p}\right\}$, where $\lambda_{i p}$ is $i$ th eigenvalue of matrix $H_{p}^{T}+H_{p}$.

According to Algorithm 3 and condition (61), we can know that the unique solution $P>0$ of Riccati equation satisfies

$$
\begin{gathered}
P\left(A+\frac{1}{2} \lambda_{i p} \kappa B K\right)^{T}+\left(A+\frac{1}{2} \lambda_{i p} \kappa B K\right) P \\
=-Q+P B B^{T} P-\lambda_{i p} \kappa P C^{T} C P \leq-Q
\end{gathered}
$$

form which we can obtain the following inequality:

$$
\begin{aligned}
& (I \otimes P)\left(I \otimes A+\frac{1}{2} \Lambda_{p} \otimes(\kappa B K)\right)^{T} \\
& \quad+\left(I \otimes A+\frac{1}{2} \Lambda_{p} \otimes(\kappa B K)\right)(I \otimes P) \leq-I \otimes Q<0 .
\end{aligned}
$$




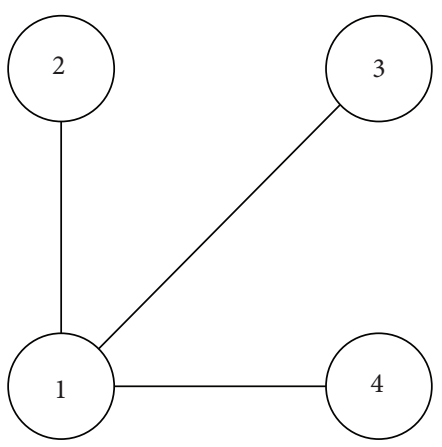

(a) $G_{1}$

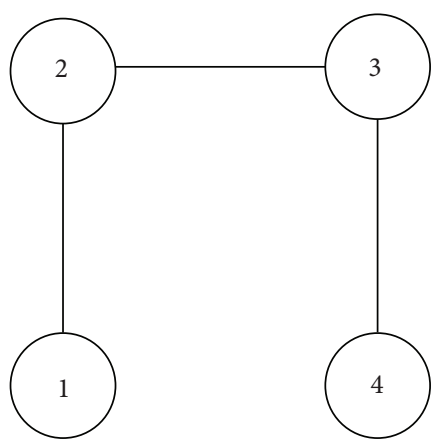

(b) $G_{2}$

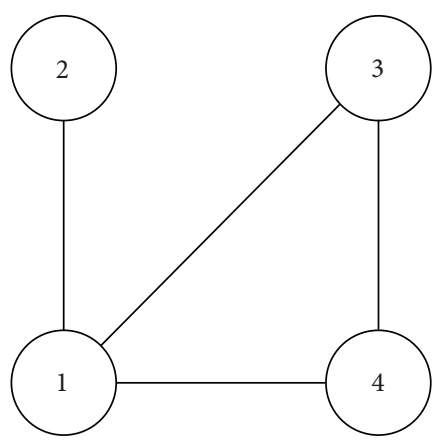

(c) $G_{3}$

Figure 1: Three interconnection topology graphs.

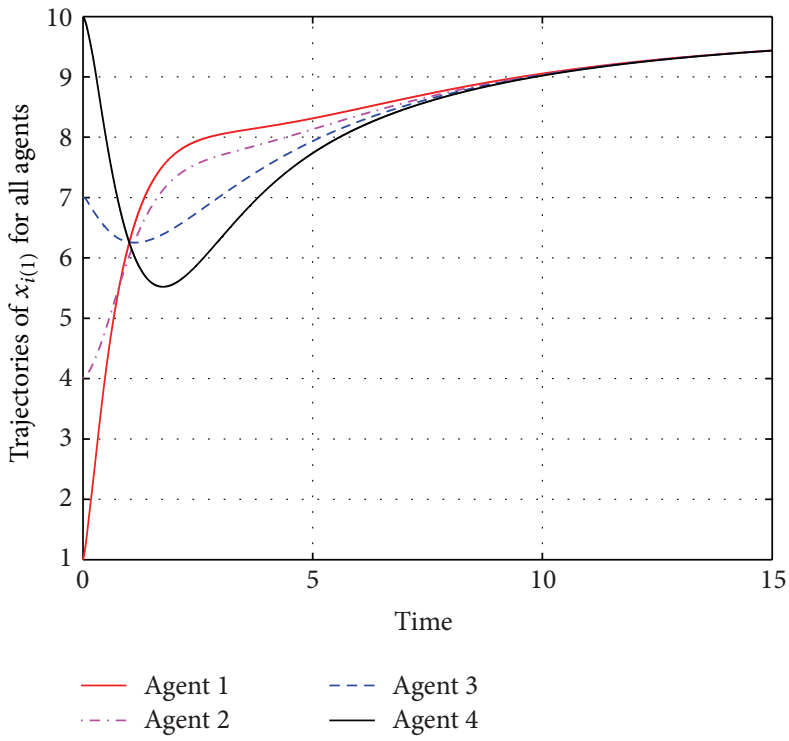

(a)

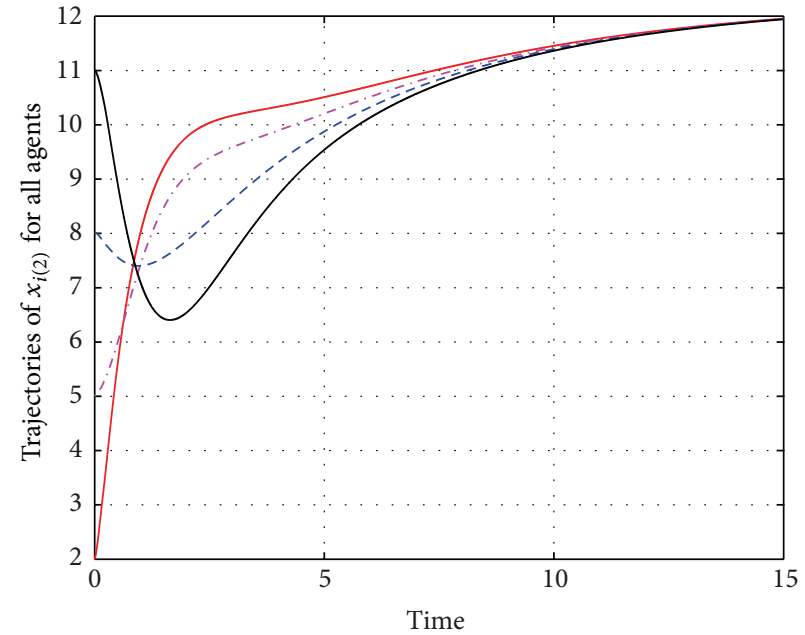

$\begin{array}{lll}\text { - Agent } 1 & -- & \text { Agent } 3 \\ -- & \text { Agent } 2 & \text { - }\end{array}$

(b)

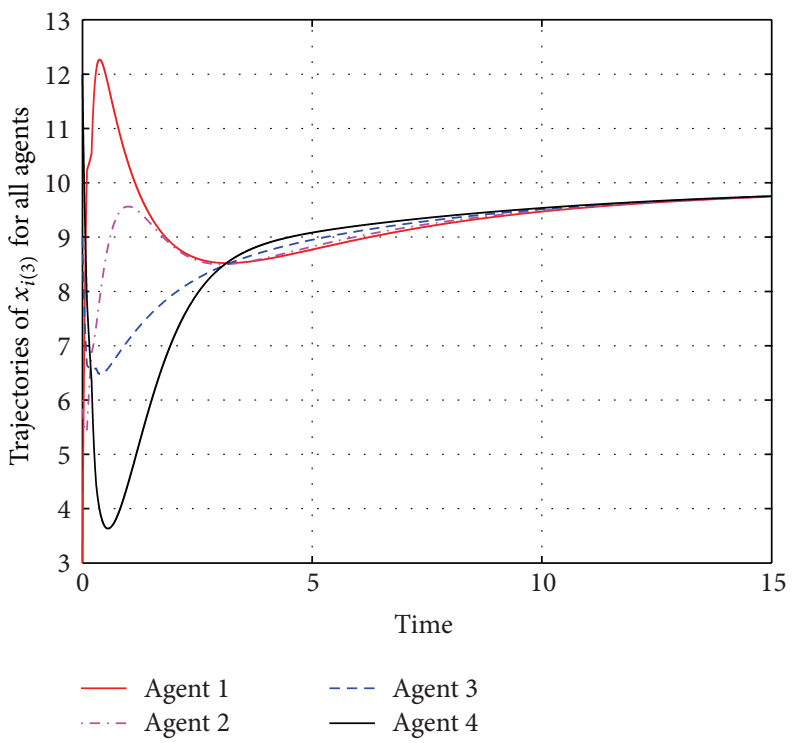

(c)

FiguRE 2: Trajectories $x_{i(j)}(t)(j=1,2,3)$ of four agents. 


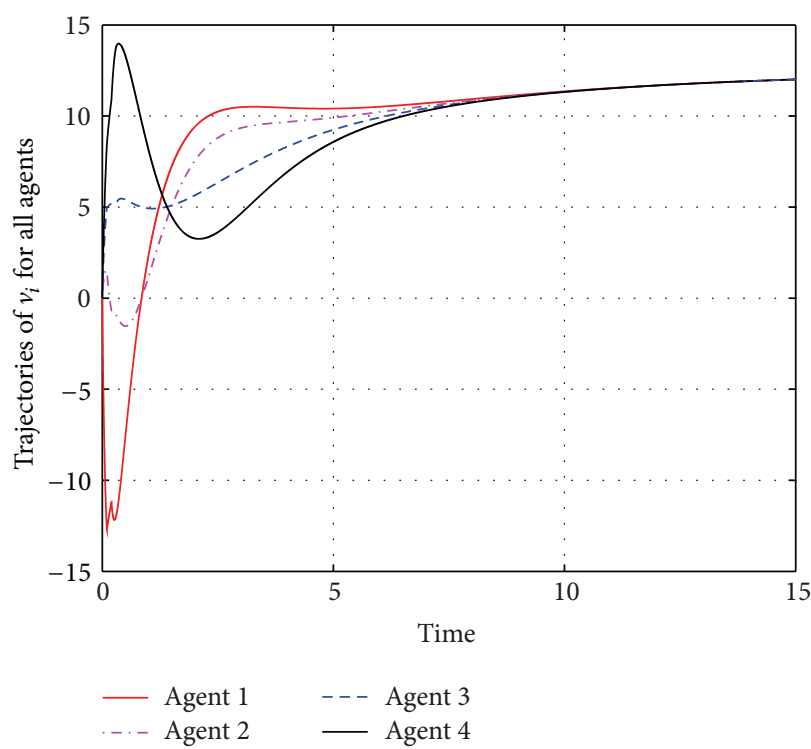

(a)

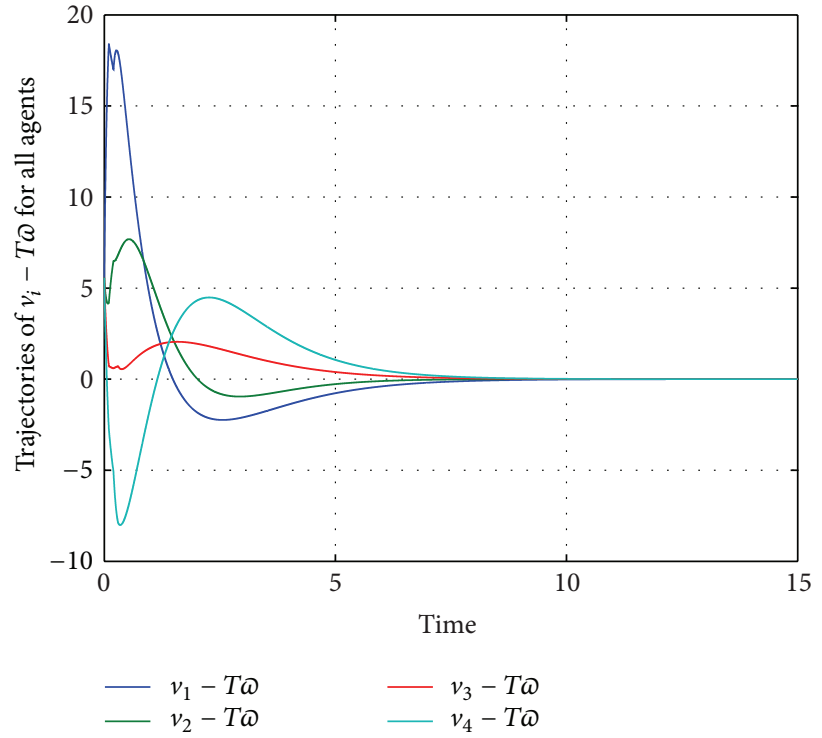

(b)

FIgURE 3: Trajectories $v_{i}^{(j)}(t)$ and $v_{i}(t)-T \omega(t)$ of four agents.

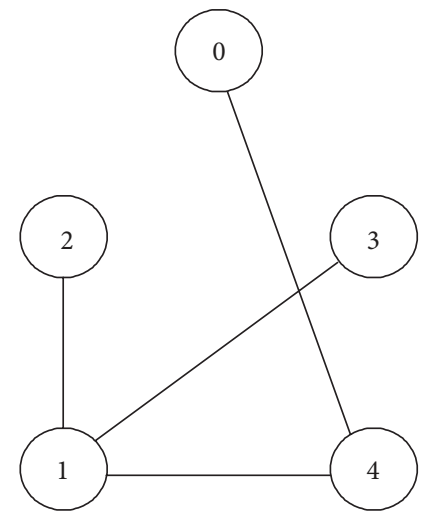

(a) $G_{1}$

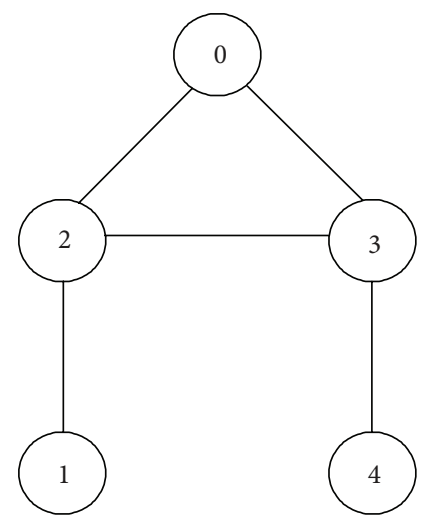

(b) $G_{2}$

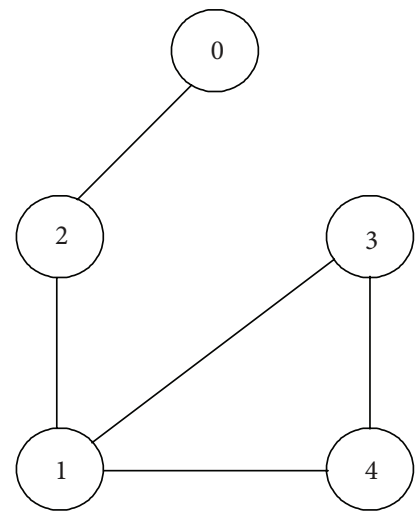

(c) $G_{3}$

FIGURE 4: Three interconnection topology graphs.

By pre- and postmultiplying (63) with $U_{p} \otimes I$ and its transpose, respectively, we have

$$
\begin{aligned}
(I & \otimes P)\left(I \otimes A+\frac{1}{2}\left(H_{p}^{T}+H_{p}\right) \otimes(\kappa B K)\right)^{T} \\
& +\left(I \otimes A+\frac{1}{2}\left(H_{p}^{T}+H_{p}\right) \otimes(\kappa B K)\right)(I \otimes P) \leq-I \otimes Q<0 .
\end{aligned}
$$

Since $P B K$ is a symmetric matrix, we know that the following inequality holds:

$$
\begin{aligned}
(I \otimes & P)\left(I \otimes A+H_{p} \otimes(\kappa B K)\right)^{T} \\
& +\left(I \otimes A+H_{p} \otimes(\kappa B K)\right)(I \otimes P)
\end{aligned}
$$

$$
\begin{aligned}
= & (I \otimes P)\left[I \otimes A+\frac{1}{2}\left(H_{p}^{T}+H_{p}\right) \otimes(\kappa B K)\right]^{T} \\
& +\left[I \otimes A+\frac{1}{2}\left(H_{p}^{T}+H_{p}\right) \otimes(\kappa B K)\right](I \otimes P) \\
\leq & -I \otimes Q<0 .
\end{aligned}
$$

Similarly, as the proof of the stability of system (29) in the proof of Theorem 6 , we can prove that the error system $\dot{\eta}=$ $\widehat{F}_{\sigma(t)} \eta$ is stable.

\section{Simulation Example}

The multiagent system contains four agents with each one modeled by the following linear dynamics: 


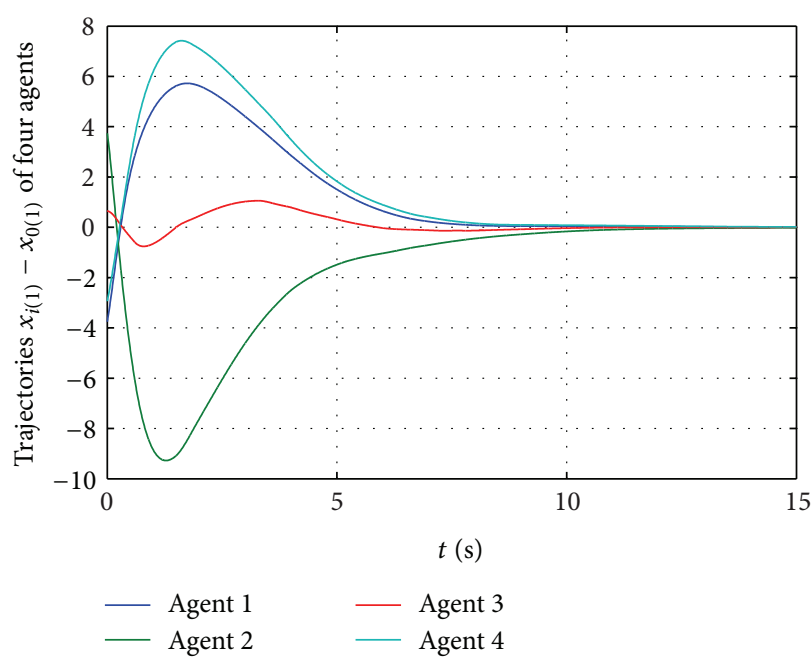

(a)

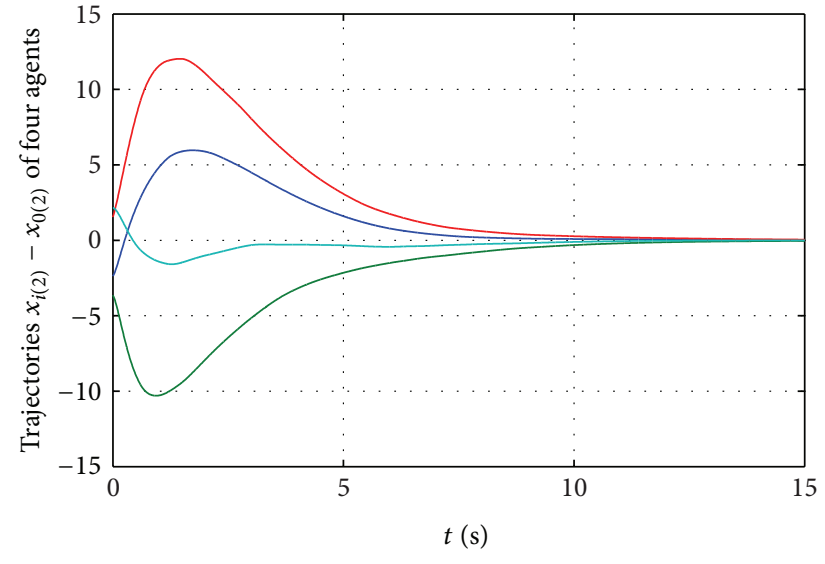

$\begin{array}{ll}\text { - Agent } 1 & - \text { Agent } 3 \\ \text { Agent } 2 & \text { Agent } 4\end{array}$

(b)

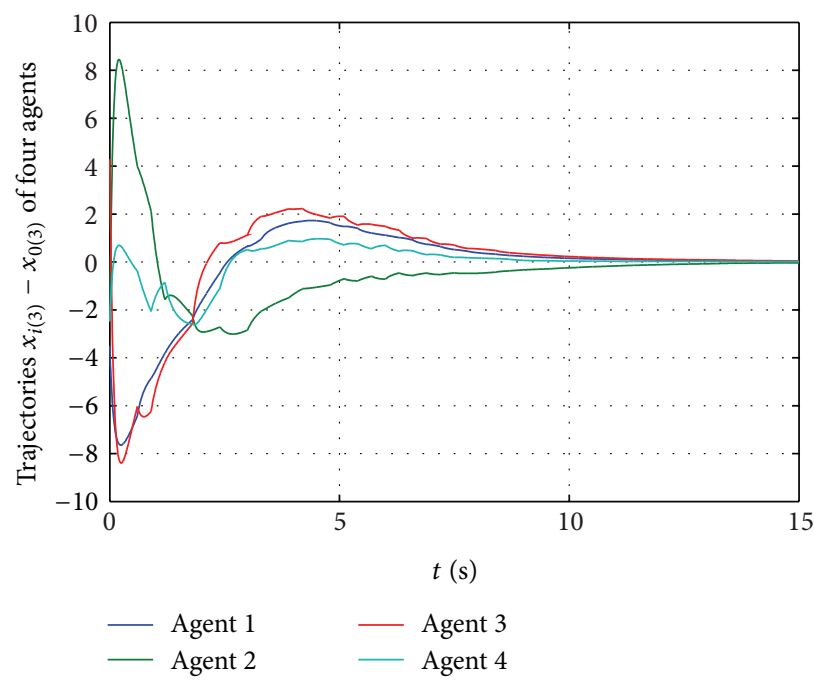

(c)

FIGURE 5: Trajectories $x_{i(j)}(t)-x_{0(j)}(j=1,2,3)$ of four agents.

$$
\begin{aligned}
& \dot{x}_{i}(t)=\left[\begin{array}{lll}
-2.1622 & 1.1469 & 0.6921 \\
-2.0497 & 0.9585 & 0.8159 \\
-1.9817 & 1.1558 & 0.5047
\end{array}\right] x_{i}(t)+\left[\begin{array}{l}
0 \\
0 \\
1
\end{array}\right] u_{i}(t), \\
& y_{i}(t)=\left[\begin{array}{lll}
1 & 0 & 0 \\
0 & 1 & 0
\end{array}\right] x_{i}(t) .
\end{aligned}
$$

The interconnection topologies are arbitrarily switched with period 0.1 s among three graphs $\mathscr{G}_{i}(i=1,2,3)$, which are shown in Figure 1. For simplicity, all nonzero weighting factors of the graphs are taken as (1).

Each agent uses the reduced-order observer-based protocol (4). Take $F=-0.8$ and $G=\left[\begin{array}{ll}0.9 & 0.1\end{array}\right]$. To solve the Sylvester equation (7), one can get $T=[9.1676-$ 4.6282 - 1.9688], which satisfies that $\left[\begin{array}{c}C \\ T\end{array}\right]$ is nonsingular. Then, we know that $Q_{1}=\left[\begin{array}{cc}1.0000 & 0 \\ 0 & 1.0000 \\ 4.6564 & -2.3508\end{array}\right]$ and $Q_{2}=$ $\left[\begin{array}{c}0 \\ 0 \\ -0.5079\end{array}\right]$. Taking a positive definite matrix $Q=4 I$, we have
$K=[3.1532-3.2555-2.7856]$ by solving the Riccati equation (8). Take $\kappa=1.0243$, which satisfies condition (31).

Let $x_{i(j)}(t)(j=1,2,3)$ denote the $j$ th component of $x_{i}$. The trajectories of $x_{i(j)}(t)$ are depicted in Figure 2, which shows that the multiagent system can achieve consensus. The trajectories of $v_{i}(t)$ and $v_{i}(t)-T \omega(t)$ are depicted in Figure 3, which is also shown that $v_{i}(t) \rightarrow T \omega(t)$ as $t \rightarrow \infty$.

For simplicity, we consider the group to consist of four followers and one leader; that is, $N=4$. Assume that the interconnection topologies are arbitrarily switched among three graphs $\widehat{\mathscr{G}}_{\sigma}(i=1,2,3)$ and the communication graph given by Figure 4 . All nonzero weighting factors of the graphs are taken as (1).

Take $\kappa=1.86$, which satisfies condition (61). All other parameters used in protocol (50) are chosen as aforementioned. The trajectories of $x_{i(j)}-x_{0(j)}, j=1,2,3$, are depicted in Figure 5, which shows that the follower agents can track the leader agent. 


\section{Conclusion}

In this paper, we investigate the consensus problem for a group of agents with high-dimensional linear coupling dynamics under undirected switching interaction topology. We propose a strict proof of main result in [35] based on the Jordan decomposition method and generalize the proposed protocol to solve the consensus problem under undirected switching interconnection topology. To solve the leaderfollowing consensus problem, we propose a neighbor-based track law for each following agent with a little simple modification to the reduced-order observer-based consensus protocol. The control parameters used in protocol can be constructed by solving the Riccati equation and the Sylvester equation. The parameter-dependent common Lyapunov function method is involved to analyze the consensus problems under undirected switching topology. Since common Lyapunov function method is conservative, the less conservative method should be probed. Of course, more generalized and interesting cases, such as switching directed interaction topology, random interaction topology, jointly connected convergence condition, and the effect of time delays arising in the communication between agents, will be investigated in our future work.

\section{Acknowledgments}

This work was supported by the National Nature Science Function of China under Grant nos. 61074123 and 61174063 and the open project of State Key Laboratory of Industrial Control Technology in Zhejiang University, China, under Grant no. ICT1218.

\section{References}

[1] A. Jadbabaie, J. Lin, and A. S. Morse, "Coordination of groups of mobile autonomous agents using nearest neighbor rules," IEEE Transactions on Automatic Control, vol. 48, no. 6, pp. 988-1001, 2003.

[2] T. Vicsek, A. Czirk, E. Ben-Jacob, I. Cohen, and O. Shochet, "Novel type of phase transition in a system of self-driven particles," Physical Review Letters, vol. 75, no. 6, pp. 1226-1229, 1995.

[3] N. Corson, M. A. Aziz-Alaoui, R. Ghnemat, S. Balev, and C. Bertelle, "Modeling the dynamics of complex interaction systems: from morphogenesis to control," International Journal of Bifurcation and Chaos, vol. 22, no. 2, Article ID 1250025, 20 pages, 2012.

[4] W. Ren and R. W. Beard, Distributed Consensus in Multivehicle Cooperative Control: Theory and Applications, Springer, Berlin, Germany, 2008.

[5] W. Yu, G. Chen, and M. Cao, "Some necessary and sufficient conditions for second-order consensus in multi-agent dynamical systems," Automatica, vol. 46, no. 6, pp. 1089-1095, 2010.

[6] F. Xiao and L. Wang, "Consensus problems for highdimensional multi-agent systems," IET Control Theory and Applications, vol. 1, no. 3, pp. 830-837, 2007.

[7] R. Olfati-Saber, A. A. Fax, and R. M. Murray, "Consensus and cooperation in networked multi-agent systems," Proceedings of the IEEE, vol. 95, no. 1, pp. 215-233, 2007.
[8] Y. Hong, J. Hu, and L. Gao, "Tracking control for multiagent consensus with an active leader and variable topology," Automatica, vol. 42, no. 7, pp. 1177-1182, 2006.

[9] J. Hu and Y. Hong, "Leader-following coordination of multiagent systems with coupling time delays," Physica A, vol. 374, no. 2, pp. 853-863, 2007.

[10] W. Ren, "On consensus algorithms for double-integrator dynamics," IEEE Transactions on Automatic Control, vol. 53, no. 6, pp. 1503-1509, 2008.

[11] P. Lin and Y. Jia, "Further results on decentralised coordination in networks of agents with second-order dynamics," IET Control Theory \& Applications, vol. 3, no. 7, pp. 957-970, 2009.

[12] W. Ren, K. L. Moore, and Y. Chen, "High-order and model reference consensus algorithms in cooperative control of multivehicle systems," ASME Journal of Dynamic Systems, Measurement, and Control, , vol. 129, no. 5, pp. 678-688, 2007.

[13] F. Jiang and L. Wang, "Consensus seeking of high-order dynamic multi-agent systems with fixed and switching topologies," International Journal of Control, vol. 83, no. 2, pp. 404420, 2010.

[14] W. Ren and R. W. Beard, "Consensus seeking in multiagent systems under dynamically changing interaction topologies," IEEE Transactions on Automatic Control, vol. 50, no. 5, pp. 655661, 2005.

[15] Y. Hong and X. Wang, "Multi-agent tracking of a highdimensional active leader with switching topology," Journal of Systems Science \& Complexity, vol. 22, no. 4, pp. 722-731, 2009.

[16] L. Gao, Y. Tang, W. Chen, and H. Zhang, "Consensus seeking in multi-agent systems with an active leader and communication delays," Kybernetika, vol. 47, no. 5, pp. 773-789, 2011.

[17] W. Ni and D. Cheng, "Leader-following consensus of multiagent systems under fixed and switching topologies," Systems \& Control Letters, vol. 59, no. 3-4, pp. 209-217, 2010.

[18] L. Gao, J. Zhang, and W. Chen, "Second-order consensus for multiagent systems under directed and switching topologies," Mathematical Problems in Engineering, vol. 2012, Article ID 273140, 21 pages, 2012.

[19] R. Yamapi, H. G. Enjieu Kadji, and G. Filatrella, "Stability of the synchronization manifold in nearest neighbor nonidentical van der Pol-like oscillators," Nonlinear Dynamics, vol. 61, no. 1-2, pp. 275-294, 2010.

[20] Y. Chen, J. Lü, F. Han, and X. Yu, "On the cluster consensus of discrete-time multi-agent systems," Systems \& Control Letters, vol. 60, no. 7, pp. 517-523, 2011.

[21] J. Zhao, M. A. Aziz-Alaoui, and C. Bertelle, "Cluster synchronization analysis of complex dynamical networks by input-tostate stability," Nonlinear Dynamics, vol. 70, no. 2, pp. 1107-1115, 2012.

[22] Y. Sun, "Mean square consensus for uncertain multiagent systems with noises and delays," Abstract and Applied Analysis, vol. 2012, Article ID 621060, 18 pages, 2012.

[23] H. Li, "Observer-type consensus protocol for a class of fractional-order uncertain multiagent systems," Abstract and Applied Analysis, vol. 2012, Article ID 672346, 18 pages, 2012.

[24] X.-R. Yang and G.-P. Liu, "Necessary and sufficient consensus conditions of descriptor multi-agent systems," IEEE Transactions on Circuits and Systems I, vol. 59, no. 11, pp. 2669-2677, 2012.

[25] F. Sun, Z.-H. Guan, X.-S. Zhan, and F.-S. Yuan, "Consensus of second-order and high-order discrete-time multi-agent systems with random networks," Nonlinear Analysis: Real World Applications, vol. 13, no. 5, pp. 1979-1990, 2012. 
[26] U. Münz, A. Papachristodoulou, and F. Allgöwer, "Consensus in multi-agent systems with coupling delays and switching topology," IEEE Transactions on Automatic Cotrol, vol. 56, no. 12, pp. 2976-2982, 2011.

[27] X. Wang, Y. Hong, J. Huang, and Z.-P. Jiang, "A distributed control approach to a robust output regulation problem for multi-agent linear systems," IEEE Transactions on Automatic Control, vol. 55, no. 12, pp. 2891-2895, 2010.

[28] Y. Su and J. Huang, "Cooperative output regulation of linear multi-agent systems," IEEE Transactions on Automatic Control, vol. 57, no. 4, pp. 1062-1066, 2012.

[29] Y. Hong, G. Chen, and L. Bushnell, "Distributed observers design for leader-following control of multi-agent networks," Automatica, vol. 44, no. 3, pp. 846-850, 2008.

[30] L. Gao, X. Zhu, and W. Chen, "Leader-following consensus problem with an accelerated motion leadera," International Journal of Control, Automation and Systems, vol. 10, no. 5, pp. 931-939, 2012.

[31] S. Bowong and R. Yamapi, "Adaptive observer based synchronization of a class of uncertain chaotic systems," International Journal of Bifurcation and Chaos in Applied Sciences and Engineering, vol. 18, no. 8, pp. 2425-2435, 2008.

[32] Z. Li, Z. Duan, G. Chen, and L. Huang, "Consensus of multiagent systems and synchronization of complex networks: a unified viewpoint," IEEE Transactions on Circuits and Systems I, vol. 57, no. 1, pp. 213-224, 2010.

[33] H. Zhang, F. L. Lewis, and A. Das, "Optimal design for synchronization of cooperative systems: state feedback, observer and output feedback," IEEE Transactions on Automatic Control, vol. 56, no. 8, pp. 1948-1952, 2011.

[34] L. Gao, X. Zhu, W. Chen, and H. Zhang, "Leader-following consensus of linear multi-agent systems with state-observer under switching topologies," Mathematical Problems in Engineering, vol. 2013, Article ID 873140, 12 pages, 2013.

[35] Z. Li, X. Liu, P. Lin, and W. Ren, "Consensus of linear multiagent systems with reduced-order observer-based protocols," Systems \& Control Letters, vol. 60, no. 7, pp. 510-516, 2011.

[36] J. H. Seo, H. Shim, and J. Back, "Consensus of high-order linear systems using dynamic output feedback compensator: low gain approach," Automatica, vol. 45, no. 11, pp. 2659-2664, 2009.

[37] Z. Li, Z. Duan, and G. Chen, "Dynamic consensus of linear multi-agent systems," IET Control Theory \& Applications, vol. 5, no. 1, pp. 19-28, 2011.

[38] W. Dong, "Distributed observer-based cooperative control of multiple nonholonomic mobile agents," International Journal of Systems Science, vol. 43, no. 5, pp. 797-808, 2012.

[39] R. A. Horn and C. R. Johnson, Matrix Analysis, Cambridge University Press, New York, NY, USA, 1985.

[40] C. Chen, Linear System Theory and Design, Oxford University Press, New York, NY, USA, 1999.

[41] W. M. Wonham, Linear Multivariable Control, Springer, New York, NY, USA, 1985. 


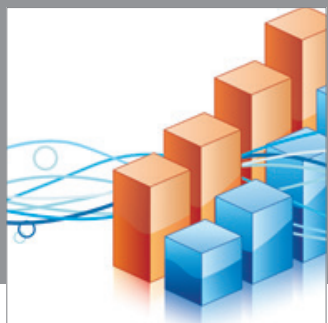

Advances in

Operations Research

mansans

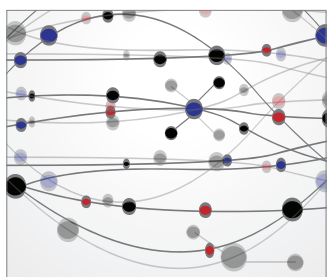

The Scientific World Journal
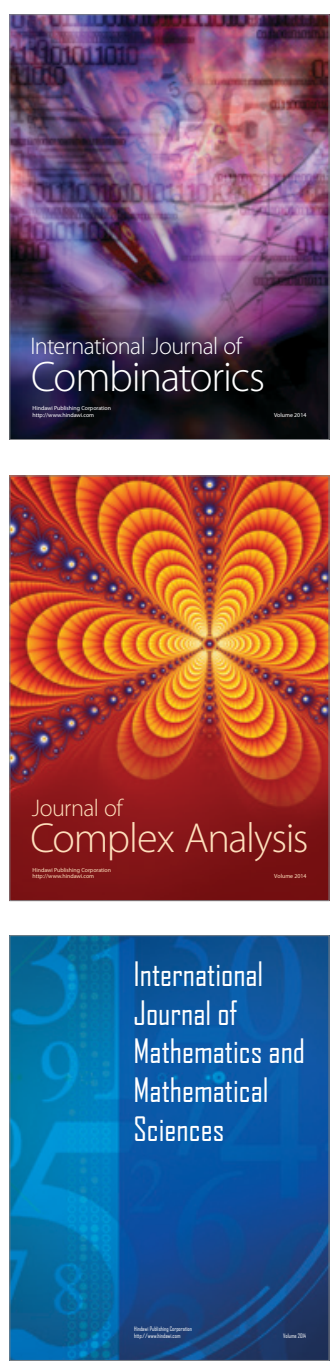
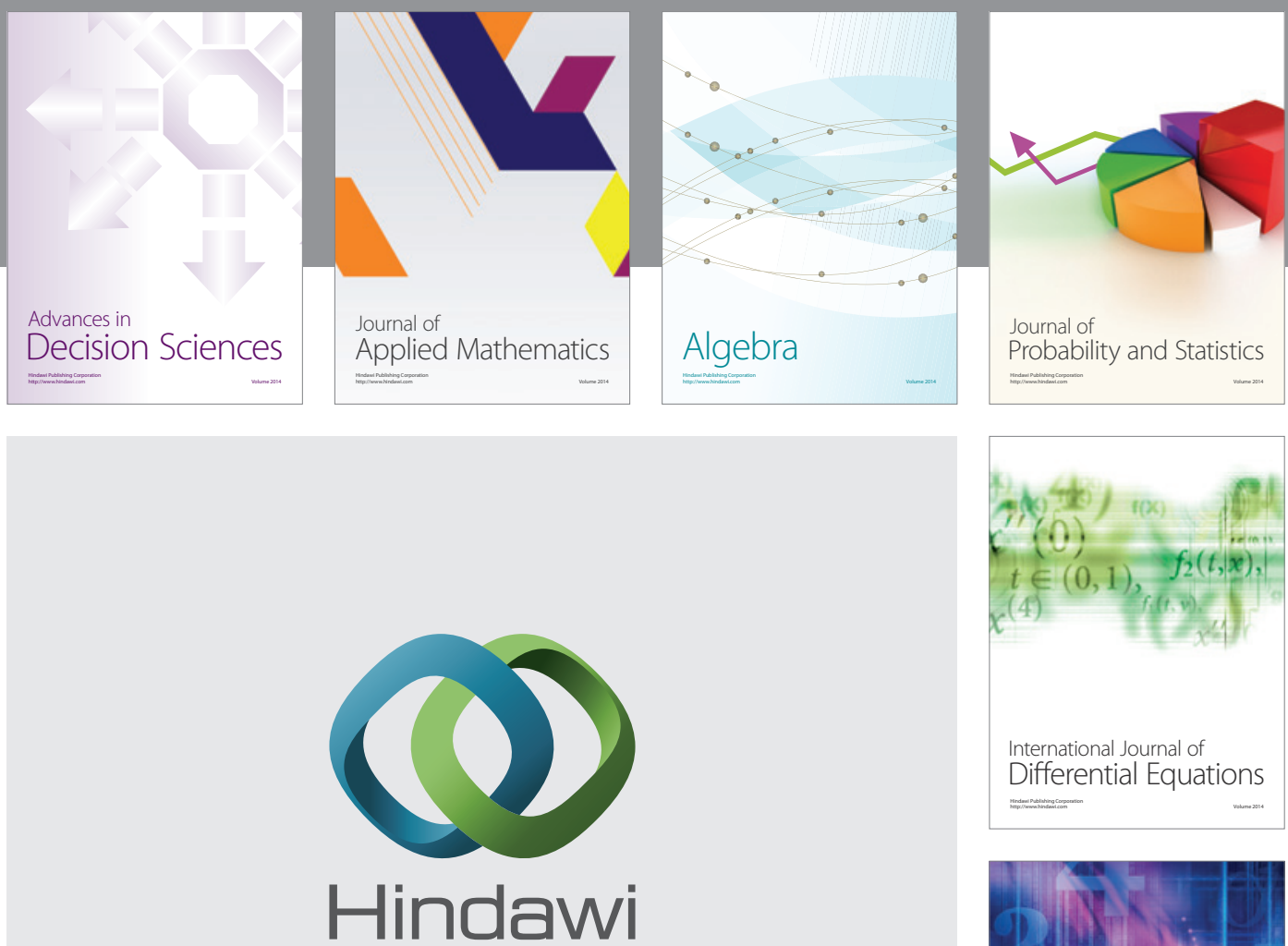

Submit your manuscripts at http://www.hindawi.com
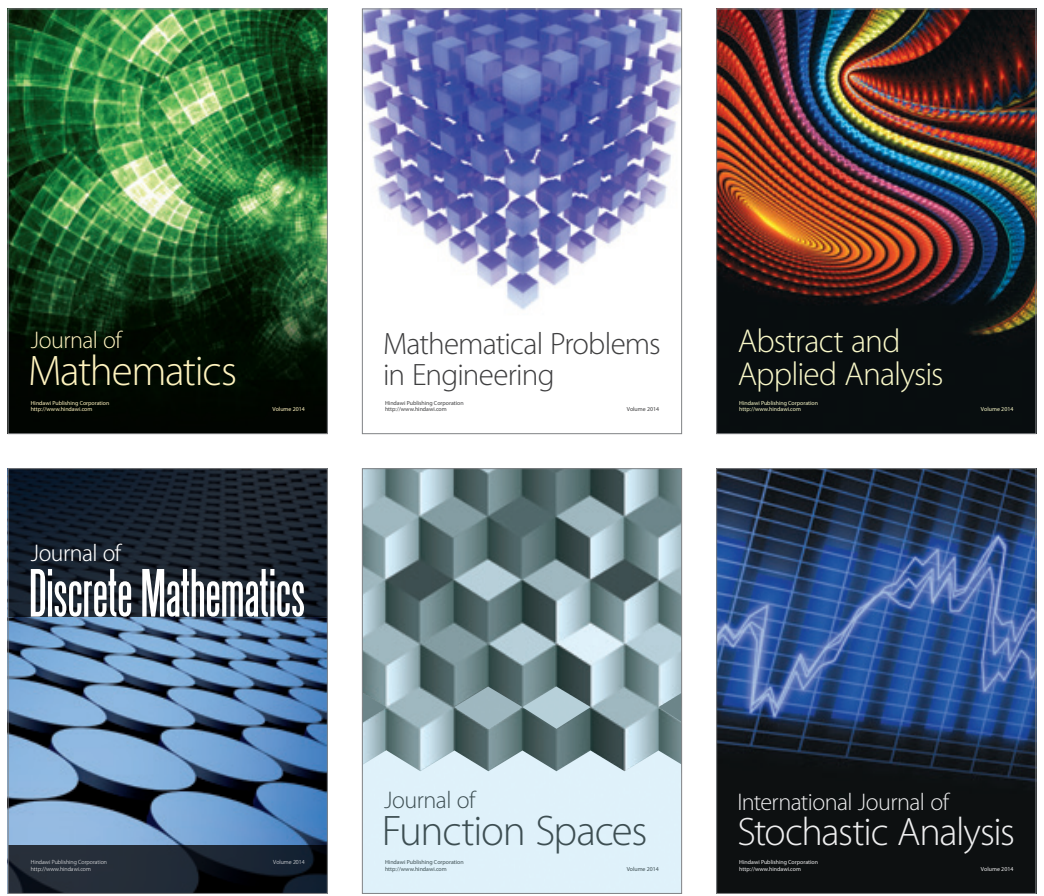

Journal of

Function Spaces

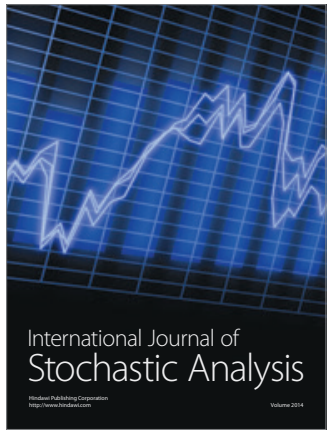

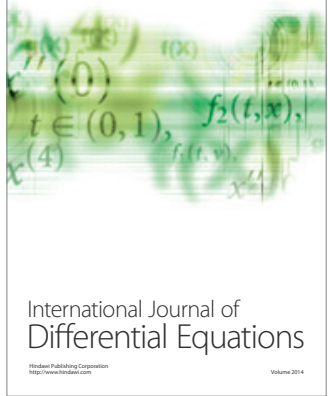
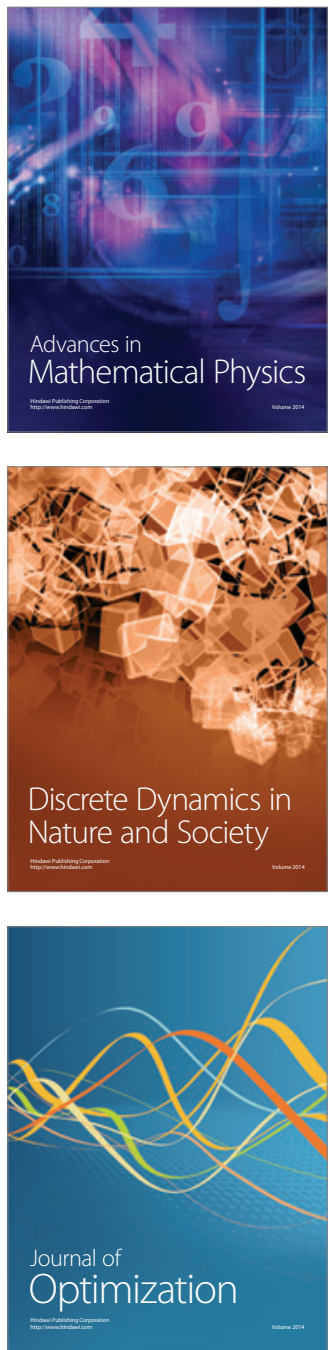